\title{
THE BEHAVIOURALLY DISTURBED PATIENT WITH HIV/AIDS
}

\author{
Tom H Boyles, $M A, B M B C h, M R C P, D T M \& H, M D$ \\ Madwaleni Hospital, Eastern Cape, and Division of Clinical Pharmacology, University of Cape Town \\ John A Joska, MMed (Psych), FCPsych (SA) \\ Department of Psychiatry and Mental Health, University of Cape Town and Groote Schuur Hospital, Cape Town
}

While HIV invades the brain early in the course of HIV infection, ${ }^{1}$ severe mental illness probably only occurs later in the disease. ${ }^{2}$ In many instances this may be the first presentation of a psychiatric illness in a younger person. In addition, the clinical syndrome may include manic and/or psychotic features, together with neurocognitive disturbance. These patients are at risk of secondary opportunistic infections or other features of systemic immunocompromise which may cause or confound the clinical picture.

\section{A CLINICAL CASE}

A 30-year-old woman presented to a rural district hospital in the Eastern Cape. She was unable to give any history. Her family said she had recently returned from Cape Town. She had seemed normal on the day she arrived home but since then had become increasingly confused but with no specific complaints. Her patient records confirmed that she had recently completed 6 months of TB treatment and had started antiretrovirals (ARVs), comprising stavudine (D4T), lamivudine (3TC) and efavirenz, 2 months previously. Her CD4 nadir was 180 cells $/ \mu$ l.

On examination she was apyrexial, with a pulse rate of $120 / \mathrm{min}$ and a blood glucose level of $8.0 \mathrm{mmol} / \mathrm{l}$. There was no meningism or clear focal neurological deficit, so lumbar puncture was not performed. On mental state examination she was found to be agitated and aggressive, with loud and incoherent speech; it was not possible to elicit delusions, but she appeared to be hallucinating. She gave appropriate answers to some simple questions and was orientated to month and year but not to day or date. A working diagnosis of psychosis secondary to HIV was made.

\section{CLINICAL PRESENTATION}

Where history from the patient is limited, a collateral history is essential.

- There are potentially multiple contributing causes, such as HIV itself, drugs including ARVs, opportunistic infections including tuberculosis or a primary psychotic disorder.

- It is critical to exclude delirium through careful clinical evaluation and targeted special investigations.

The patient was sedated with haloperidol and diazepam and admitted for further investigation. The following day she remained aggressive and confused. Efavirenz was stopped but D4T and 3TC were continued. Over the next week there was gradual improvement in her mental state. She became oriented to day and place, but complained of seeing people who were coming to steal her medication. Collateral history from her mother was that she had no known previous psychiatric history and that the hallucinations had started during her father's funeral a week before admission.

The results of basic blood tests were normal other than C-reactive protein (CRP) $63.4 \mathrm{mg} / \mathrm{l}$ and platelets $67 \mathrm{x}$ $10^{9} / 1$.

\section{INVESTIGATION}

- In a case where severe behavioural disturbance is present in a setting of severe immunocompromise, investigations should include basic blood work-up, as well as lumbar puncture and a computed tomography scan of the brain.

- If confusion is prominent and/or the patient has a headache and/or fever, it is prudent to request polymerase chain reaction (PCR) testing of the cerebrospinal fluid for cytomegalovirus, herpes simplex virus, Epstein-Barr virus and JC virus.

After 1 week, the diagnosis of psychosis secondary to HIV was confirmed by a doctor at a tertiary level psychiatric unit. The recommendation was to wean the patient off haloperidol and replace it with risperidone. Risperidone is not available at level 1 district hospitals, so haloperidol $2.5 \mathrm{mg} 3$ times daily was continued. Nevirapine was introduced after 10 days. She was discharged a week later.

Two weeks later, having defaulted her follow-up appointment, she presented with what the admitting doctor described as 'aggressive and psychotic behaviour. He increased the haloperidol dose to $5 \mathrm{mg}$ twice 
a day plus diazepam $5 \mathrm{mg}$ at night. She claimed that her family were trying to kill her. She was prescribed fluphenazine $12.5 \mathrm{mg}$ as an intramuscular injection and over the next week she settled and regained orientation to time, person and place. Five days later, after much discussion with her family, she was discharged with a review date in 1 month.

One month later, the clinical presentation recurred. She was sedated with haloperidol and lorazepam and after consultation with an HIV psychiatrist valproate $200 \mathrm{mg}$ bd was added. It had emerged that prominent mood symptoms were present, namely irritability and expansiveness. The persecutory delusions were paired with grandiosity. Over the next week she gradually improved and regained her orientation to time and place. Valproate was increased to $400 \mathrm{mg}$ bd and nevirapine was changed back to efavirenz on the basis that stopping the efavirenz had had no impact on her mental state and there is a potential drug-drug interaction between valproate and nevirapine.

A week later, the patient was feeling well with no psychotic symptoms and was fully orientated. She was discharged with a prescription for valproate $400 \mathrm{mg}$ bd. She has now been followed up regularly for 4 months. She remains completely well and is adherent to both her valproate and ARVs.

\section{PSYCHOTROPICS}

Haloperidol is safe to use in this setting with the drawback of a high potential for extrapyramidal side-effects; atypical antipsychotics, such as risperidone $0.5-2 \mathrm{mg} 2 \times$ daily or quetiapine 50 - $200 \mathrm{mg} 2 \times$ daily, if available, may be better.

- In the short term, lorazepam 2 - 4 mg 8-hourly (or oxazepam if liver impairment is present) may be used for sedation.

- If manic symptoms are a prominent feature, consider using valproate $300 \mathrm{mg} 2 \times$ daily, increasing to $600 \mathrm{mg} 2 \times$ daily; use lower doses and monitor liver function tests if liver impairment is present.

\section{DISCUSSION}

Neuropsychiatric presentations in late-stage HIV require clinicians to carefully exclude a range of possible contributory causes. One case series reported a $2 \%$ incidence of new-onset psychosis in patients presenting with an AIDS-defining diagnosis. Of these, 50\% had an underlying infective or metabolic cause while 50\% were thought to have an HIV-related psychosis. ${ }^{3}$

Infectious causes may be due to bacteria, mycobacteria, viruses, fungi or spirochaetes and may form part of an immune reconstitution inflammatory syndrome (IRIS) if occurring after the onset of ARV medication. In the above case, the absence of fever and headache weighed against this possibility.
Medication-related causes of psychosis also occur. These include $A R V s,{ }^{4}$ antituberculosis drugs and prednisone. Efavirenz is often implicated. A stepwise approach to removing potential drug causes is advised - treat the psychosis or mania appropriately, and if no response is seen, remove the most likely offending agent. Once the effect (or not) of this move has been appraised, an informed decision to make additional changes or switches can be made.

Primary psychiatric disorders are frequently seen in the setting of HIV infection. While bipolar disorder, mania and major depressive disorder with psychosis must all be considered, so too must the effects of psychosocial stressors such as bereavement, loss, unemployment and disability. Psychotropic treatments need to be used alongside psychosocial measures, which include educating families, addressing needs for disability grants and counselling.

The management of behaviourally disturbed patients with HIV in a remote rural hospital presents many challenges. One such problem is that once the diagnosis of HIV infection is made there is a tendency to attribute multiple symptoms to the HIV itself without a full consideration of other possibilities. While the initial probability of HIV-related psychosis may be fairly high in the presence of suggestive features, a definitive diagnosis requires the exclusion of a number of other possibilities.

A similar problem is related to the treatment of all patients with psychiatric illness in this setting, namely that they are labelled a 'psych patient' without consideration of which of many underlying conditions may be responsible for their symptoms. This situation is exacerbated by lack of resources. Under these conditions the standard of care for patients with features of psychosis is monthly injectable antipsychotics with reliance on the patient and relatives to ensure adherence. Patients with features of depression are similarly prescribed low-dose amitriptyline, often without a full explanation of time course or dose of treatment required. With more complex interventions being beyond the scope of the service, it is perhaps understandable that little attention is paid to the exact diagnosis. However, despite these drawbacks it is important that all patients are seen by a doctor at the district hospital and that complex cases be referred to the tertiary centre for an opinion and a follow-up plan before being referred back to peripheral clinics.

\section{REFERENCES}

1. McArthur JC, Brew BJ, Nath A. Neurological complications of HIV infection. Lancet Neurol 2005; 4(9): 543-555.

2. Dube B, Benton T, Cruess DG, Evans DL. Neuropsychiatric manifestations of HIV infection and AIDS. J Psychiatry Neurosci 2005; 30(4): 237-246.

3. Alciati A, Fusi A, d'Arminio MA, Coen M, Ferri A, Mellado C. New-onset delusions and hallucinations in patients infected with HIV. J Psychiatry Neurosci 2001; 26(3): 229-234.

4. Foster $R$, Olajide $D$, Everall IP. Antiretroviral therapy-induced psychosis: case report and brief review of the literature. HIV Med 2003; 4(2): 139-144. 